\title{
Desarrollo y transferencia tecnológica
}

Gabriel Sorto ${ }^{1}$

Rafael Núñez Solórzano ${ }^{2}$

\section{$\mathrm{CT} / ¿$ Existen condiciones favorables en la UNAH para la investigación aplicada en áreas de conocimiento de su competencia?}

GS/ Con respecto al tema de las condiciones favorables, sí creo que existen condiciones, especialmente sobre algunos aspectos sobre los cuales quiero hacer énfasis. Uno de ellos es el conocimiento por sí mismo y la experiencia de la UNAH. Actualmente, en la parte académica en las cátedras se dan los conocimientos que son base para desarrollar ideas creativas o innovadoras. Otro aspecto también importante son las plataformas tecnológicas, tales como las redes de internet; estas plataformas son espacios para buscar información sobre temas. Actualmente en nuestra sociedad, por ende también en la UNAH, existen muchos desafíos en los cuales tenemos que aplicar soluciones desde la ingeniería. Así, la UNAH cuenta con lo básico para desarrollar investigaciones para resolver múltiples problemas en nuestro país.

RNS/ Las condiciones cada día se vuelven más favorables en nuestro campo del conocimiento, que son las ciencias económicas y administrativas, para que podamos realizar investigación aplicada. De hecho, contamos con un instituto de investigaciones que presta servicios de diferente índole; en este podemos desarrollar nuestras investigaciones y ya tenemos algunos elementos

\footnotetext{
1 Posee una licenciatura en Ingeniería Eléctrica por la Universidad Nacional Autónoma de Honduras (UNAH) y realizó una Maestría en Gestión de Proyectos en la Universidad Católica de Honduras (UNICAH). Actualmente se desempeña como coordinador técnico de la Sección Electromecánica y Telecomunicación en la Dirección Técnica de Proyectos en la Secretaría Ejecutiva de Administración de Proyectos de Infraestructura, SEAPI-UNAH. Además, acompaño al Profesor Dennis Rivera como beneficiario de una beca sustantiva con el proyecto: "Sistema SCADA-UNAH para eficiencia energética, implementación de edificio piloto". Con los conocimientos obtenidos a partir de esta investigación aplicada llevó a cabo la creación de la plataforma de inmótica de edificios que la UNAH ha construido y sigue construyendo; por ejemplo, el Palacio de los Deportes, Edificio de Rectoría y Ciencias de la Salud, entre otros proyectos futuros, como el edificio de posgrados.
}

2 Obtuvo su licenciatura en Administración de Empresas y una Maestría en Administración de Empresas por la Universidad Nacional Autónoma de Honduras. Cursó el Diplomado en Administración de los Centros de Desarrollo Empresarial de las Mipyme; además, es especialista en diseño y gestión curricular. Actualmente se desempeña como coordinador académico de la Carrera de Administración de Empresas y asesor del Centro de Emprendedores de la Facultad de Ciencias Económicas, Administrativas y Contables de la UNAH. Ha publicado artículos sobre innovación y desarrollo empresarial. 
importantes de avance. Creemos que la Universidad ha mejorado a través de toda la infraestructura que ha creado por medio de la Dirección de Investigación Científica y Posgrado. Con los posgrados también se apoya lo que es el proceso de investigación, dado que se ha cambiado mucho la concepción que se tenía de la investigación como un requisito de graduación. Ahora tenemos toda una plataforma de apoyo a la investigación que la UNAH ofrece a través de diferentes instancias. Hemos podido ver el rol que asume la DICYP, que ya lleva 8 congresos de investigación realizados ininterrumpidamente; cada año más fortalecidos. Nuestra facultad lleva su segundo congreso de investigación en economía, administración y tecnología. Este año vamos por el tercero. También tenemos otras unidades y el surgimiento de nuevos institutos de investigación. Entonces, sí creemos que este escenario se está terminando de consolidar.

\section{$\mathrm{CT} /$ ¿Qué condiciones son necesarias para realizar proyectos de investigación aplicada, desarrollo tecnológico e innovación desde la UNAH?}

GS/ Considero que los laboratorios para pruebas de ensayo y error de los experimentos de investigación son fundamentales. En la medida que tengamos infraestructura y equipamiento para facilitar los procesos de investigación y para realizar pruebas, esto ayudará a reducir tiempos. En nuestra investigación nos ocurrió que no teníamos equipo para probar, entonces tuvimos que adquirirlo por medio de la beca que obtuvimos de la DICYP. De este modo, una vez que tuvimos el equipo, comenzamos a probar su funcionamiento. Mediante la utilización del equipo, previo proceso de selección del mejor, logramos obtener los resultados. Por tanto, en la medida que tengamos laboratorios será posible probar diferentes cosas: corroborar hipótesis, probar nuevas soluciones 0 modificar algunos aspectos técnicos. En el aspecto de infraestructura tenemos que facilitar el espacio para albergar el equipo, ya que sin las condiciones físicas necesarias el equipo puede sufrir daños. Por último, resulta imprescindible hacer uso de las nuevas tecnologías. Un problema del país es que solamente utilizamos las herramientas tecnológicas que existen, repitiendo procesos, sin probar nuevas formas de aplicación, sin innovación. La comunidad universitaria debe incentivar los procesos de utilización de las nuevas tecnologías.

RNS/ La disposición del recurso humano es esencial, es el elemento que nosotros consideramos como una "cultura de investigación", en profesores y estudiantes. Esto tiene que ser vital, porque de nada sirve que tengamos otras condiciones 0 medios como computadores, bases de datos, acceso a revistas, pero sino hay esa disposición, esos esfuerzos no se desarrollan plenamente. En nuestro 


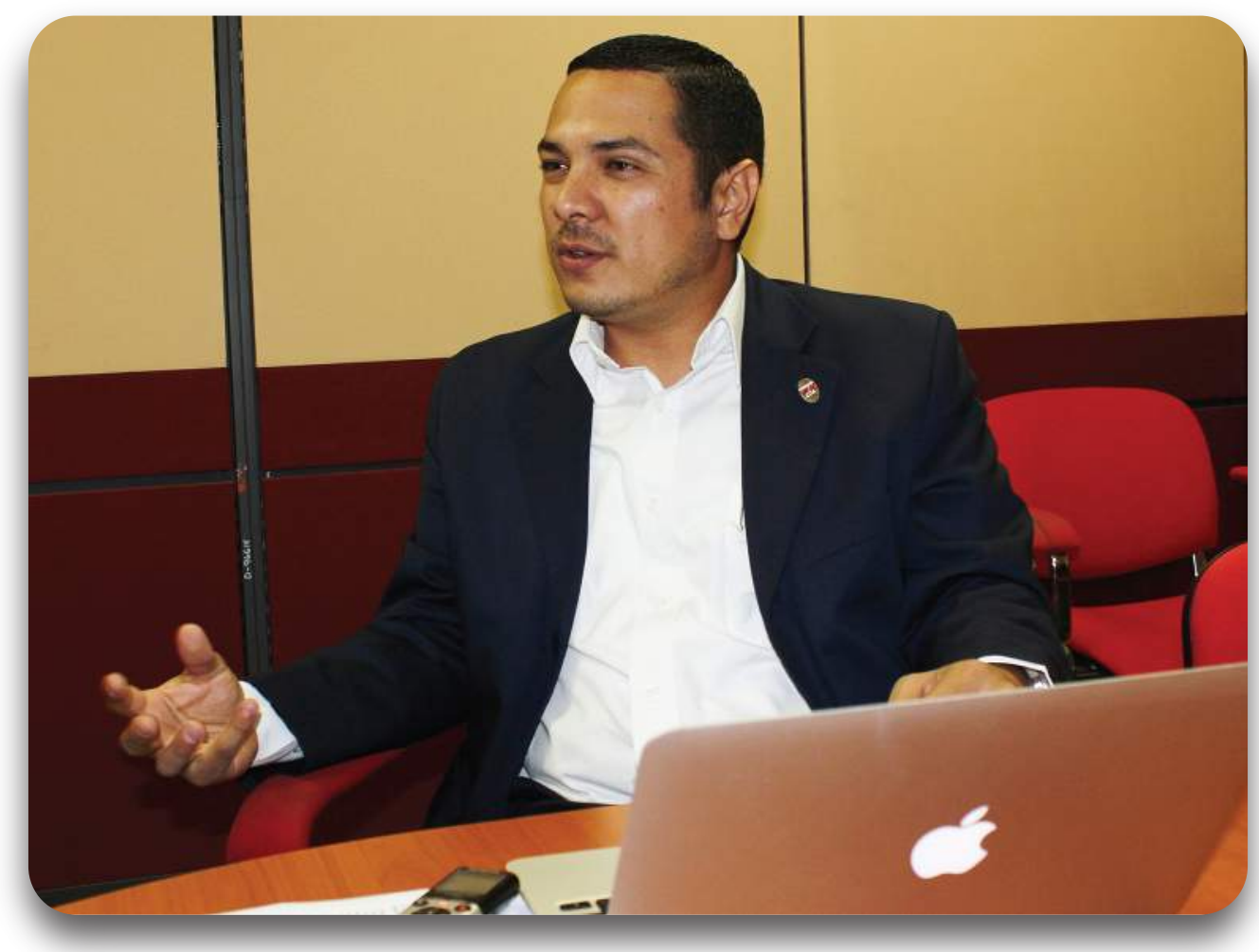

Rafael Núñez Solórzano

campo tal vez no necesitamos de un laboratorio de ensayo y error científico, en nuestro campo estamos más orientados a la investigación aplicada. Pero, sí estamos vinculados con las empresas privadas y públicas para poder investigar sobre temas económicos y administrativos, además de abordar el tema de la innovación. Hoy en día ya tenemos avances en infraestructura, como los laboratorios de cómputo que son un espacio para desarrollar parte de las investigaciones. También contamos con apoyo financiero, ya que al investigar se requiere de una inversión de recursos para distintas actividades. Aquí el apoyo de la DICYP, por medio de las becas de investigación, resulta ser una iniciativa importante para que los interesados podamos desarrollar nuestro trabajo de investigación facilitando las condiciones.

CT/ ¿Hay experiencias de proyectos científicos-tecnológicos que hayan sido transferidos a la sociedad o empresas? ¿Cuál es el valor agregado de los proyectos que ha realizado en la UNAH?

GS/ En algunos de los proyectos en los que estoy involucrado actualmente, que están 
funcionando en la UNAH, hay uno específico que quiero mencionar, es el proyecto de investigación del sistema de control y adquisición de datos para el edificio piloto de la UNAH, en el cual se espera lograr la eficiencia energética. Este proyecto contó con el apoyo de la DICYP, a través de una beca sustantiva, quien me la otorgó en conjunto con el Dr. Dennis Rivera de la Carrera de Ingeniería Eléctrica. Con este proyecto logramos desarrollar un modelo tecnológico aplicado para poder ahorrar energía por medio del control de la iluminación en horarios y sensores en tiempo real. Asimismo, hacemos un monitoreo en tiempo real de donde se puede ordenar el encendido y apagado de las luces, en el cual el operador puede tener acceso a realizar estas acciones. Entonces, el nivel de aplicabilidad en la iluminación se puede ver como algo básico, pero es parte esencial para realizar otras acciones. Gracias a este tipo de modelo ya se toma en cuenta este elemento en los proyectos que se realizan en la UNAH, incorporándose a nuevos edificios que están actualmente en construcción. Para el caso, en el Polideportivo, que es un edificio nuevo, hay dispositivos tecnológicos muy significativos, podemos decir que es uno de los edificios con un valor tecnológico de muy alto a nivel en toda la ciudad. Además, considero que es uno de los pocos edificios que tiene la integración en la parte de sistemas. Nosotros podemos controlar un generador a través del teléfono celular y el mismo sistema de iluminación de todo el edificio se puede controlar por esta vía. De igual manera, podemos estar en cualquier país y se puede tener un control del edificio, un sistema con la integración de las cámaras y sistema contra incendios, entre otros. Por tanto, el sistema de integración a nivel de estas plataformas ya se aplica en la sociedad en iniciativas como las de este edificio que posee un valor agregado. En el caso del proyecto del Polideportivo, este representaba una oportunidad para implementar la tecnología adquirida, o mejor dicho, la experiencia del conocimiento de aplicar la tecnología. Por tanto, lo que hicimos nosotros fue integrar el sistema de control de iluminación y prácticamente lo que logramos fue lograr un mayor control. En este proceso intervienen programaciones en las plataformas, conocimiento en redes, ingeniería en potencia, electrónica, automatización y telecomunicaciones. Es una mezcla y sinergias de conocimientos para llegar a ese tipo de soluciones tecnológicas.

RNS/ De hecho, oficialmente están más orientados a la sociedad, porque nosotros hemos hecho investigaciones para el gobierno en temas económicos, desarrollando indicadores económicos, abordando la temática del empleo, divisas y exportaciones, entre otros. En el caso del tema de la tecnología todavía no se ha desarrollado plenamente; pero, sí hemos realizado aportes en 
dimensiones como mejoras e innovación de procesos en algunas empresas privadas; en ese ámbito hemos trabajado como consultores o asesores, dando sugerencias o haciendo propuestas. Por tanto, necesitamos ligar la investigación y la innovación con las empresas privadas.

CT/ ¿Qué beneficios puede obtener la UNAH y el país producto de la transferencia de conocimiento científico-tecnológico?

GS/ Uno de los primeros impactos que se obtiene, por ejemplo, de este tipo de proyectos, es el ahorro en la factura eléctrica. Al tener control de los dispositivos eléctricos de diferentes maneras, se logra un ahorro energético considerable. No es lo mismo tener una bomba encendida todo el tiempo para suplir un servicio, a que este dispositivo solo lo hago cuando se le ordene hacerlo. Muchas de las infraestructuras que se construyeron en nuestra Universidad en las últimas décadas no han tenido el orden respectivo; por tal razón, desarrollar este tipo de proyectos tecnológicos y sus conocimientos aplicados, permite que la UNAH tenga un ahorro de energía y a nivel de operaciones y mantenimiento.

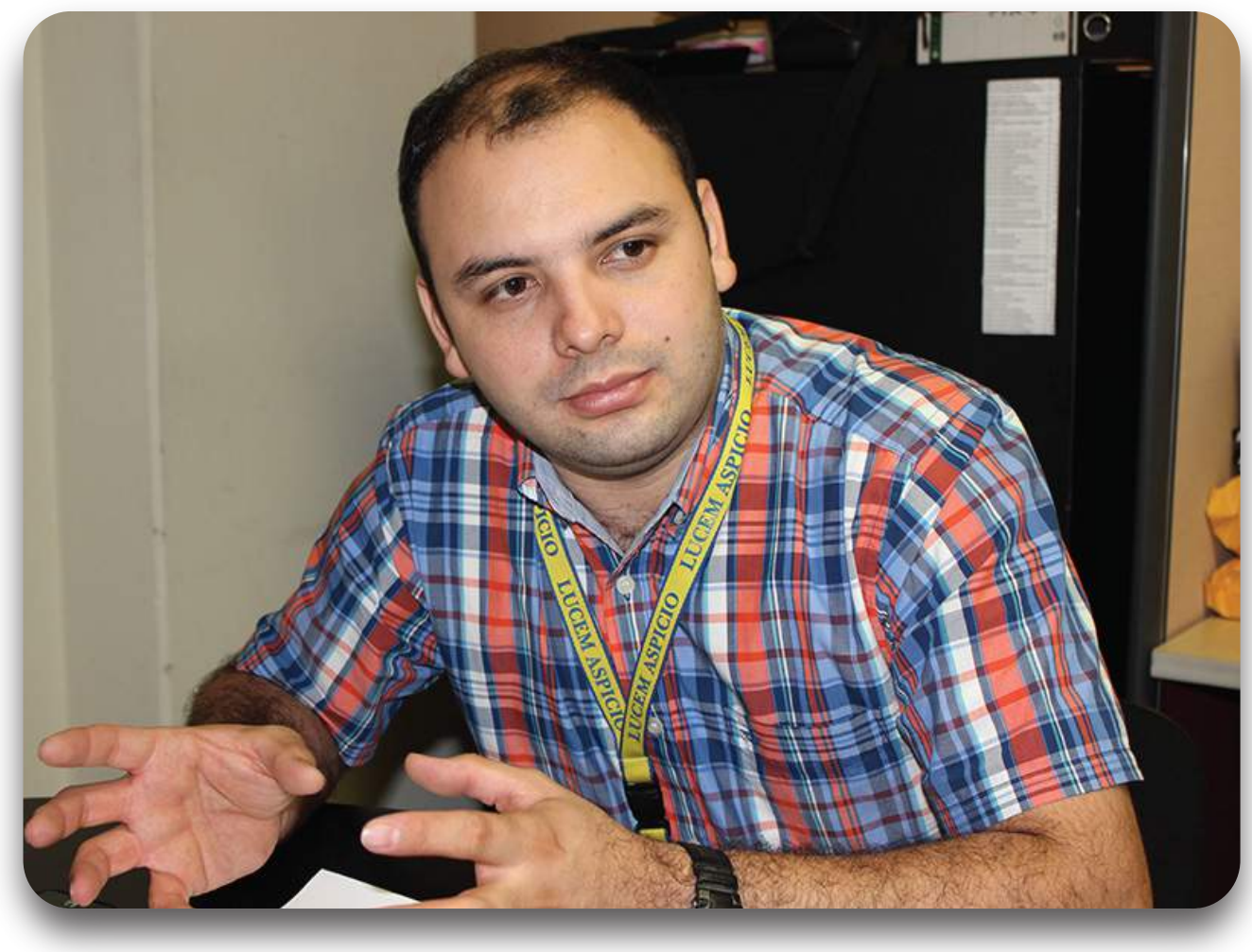

Gabriel Sorto 
Actualmente los dispositivos nos notifican cuando requieren mantenimiento y supervisión, esto permite que el personal técnico de las operaciones de mantenimiento dedique menos tiempo a realizar su trabajo, lo que implica menos horas extra, trabajo más rápido y eficiente. Esa es otra forma de beneficio de la transferencia de este tipo de conocimiento. Además, a nivel institucional la UNAH, se afianza como centro que promueve y genera innovación en su infraestructura. Otro elemento muy importante es la transferencia de conocimiento a otras personas, pues la sociedad adquiere esta información y verifica su aplicabilidad. Por tanto, la UNAH se refuerza como una institución que tiene un semillero de investigadores que realizan investigación aplicada que puede hacerse en empresas estatales o privadas.

RNS/ Desde nuestro campo hemos propuesto modelos de inversión, modelos económicos y de generación de ideas de negocios, entre otros tipos de intervenciones. Creo que nosotros podemos hacer un gran aporte desde nuestro campo del conocimiento por medio de investigaciones. Ahora la mentalidad está cambiando. Hemos entrado en un proceso en donde el profesor desarrolla investigación para poder ofrecer sus resultados a estudiantes, académicos y empresas. Nosotros tenemos algunas experiencias en las cuales los profesores han trabajado en desarrollar casos de estudio y ejemplos basados en situaciones aplicadas a nuestra realidad. Creo que la docencia y la investigación -y la integración de estas- son fundamentales para potenciar los procesos de transferencia del conocimiento.

CT/ ¿Qué actividades ha realizado para incentivar la creatividad de los estudiantes y profesores que se hayan traducido en productos, procesos y servicios susceptibles de transferencia para la solución de problemas 0 satisfacción de necesidades de la sociedad hondureña?

GS/ Conozco pocas experiencias, pero considero que una de las ventajas es que todo el conocimiento debe ser compartido. Creo que es importante darle más apoyo a las ferias vocacionales y las de innovación, en estas últimas se pueden presentar proyectos de estudiantes para que muestren sus ideas de innovación y su visión de la tecnología. Una experiencia significativa son las prácticas de los estudiantes en la Secretaría Ejecutiva de Administración de Proyectos de Infraestructura, aunque hay pocos ingenieros en la parte electromecánica, telecomunicaciones y control y automatización. Una manera de apoyar a los estudiantes es abriendo las puertas para que realicen su práctica profesional, ya que de esta forma incentivamos su creatividad, dándoles la oportunidad de 
participar en los procesos en los que estamos vinculados con la UNAH; los jóvenes que hacen la práctica tienen un entrenamiento continuo que les ayuda a adquirir conocimiento de aplicación tecnológica. Así, creamos un espacio para poner a prueba sus conocimientos y proponer soluciones a problemas encontrados.

RNS/ Nosotros venimos trabajando desde hace casi una década en el centro de emprendedores. Tratamos de cambiar el concepto de lo que es una feria. Realmente es una cultura de vida. La feria es solo un producto del centro; de esa manera, incentivamos a los jóvenes a participar y desarrollar ideas. Ahora estamos orientándonos más hacia el tema de la tecnología, como aplicaciones y software, entre otros. El último año hemos tenido avances, con el apoyo de la DICYP, en la creación de un círculo de creatividad en la Facultad de Ciencias Económicas; en tal sentido, tenemos un grupo de jóvenes trabajando y profesores en esto. Esperamos que esta iniciativa sea de beneficio para la comunidad universitaria y la sociedad en general, a través de los productos que podamos ofrecer. Otra iniciativa es el Congreso de Economía, Administración y Tecnología, en el que hemos logrado que profesores y estudiantes participen con varios temas e investigaciones.

\section{CT/ ¿Qué desafíos identifica a mediano y largo plazo para la ejecución de este tipo de proyectos en la UNAH?}

GS/ Uno de los que identifico es que necesitamos seguir investigando más. Otro es ampliar las áreas de conocimiento y aplicaciones tecnológicas, por ejemplo robótica, domótica, inmótica, microprocesadores y microcontroladores. Estos avances pueden desarrollarse bajo la creación de nuevas carreras y mejoramiento de las que existen.

RNS/ El desafío más grande es consolidar una cultura de investigación, es incorporar la investigación en muchas áreas de nuestro trabajo. Tenemos algunos avances, pero creemos que en nuestra facultad es necesario potenciar la actividad de la investigación en toda la comunidad universitaria. Todavía necesitamos llegar a más profesores y estudiantes para realizar investigación científica. Otro reto es el trabajo en conjunto con universidades de otros países, si bien es cierto que hacemos investigación, es necesario integrar las investigaciones desde una perspectiva regional. Ese es otro reto, poder hacer alianzas y convenios; lo importante es ejecutar acciones de investigación con otros países. Otro desafío es la vinculación de la universidad con las empresas para el ofrecimiento de 
soluciones. Por último, está el ofrecimiento de los espacios adecuados para ejercer la investigación, lo interesante es que ahora contamos con espacios como el Centro de Apoyo a la Tecnología y la Innovación, así como con institutos y otras instancias para la promoción de la investigación. 